OPEN ACCESS

Edited by:

Paola Marziani,

Osservatorio Astronomico di Padova

(INAF), Italy

Reviewed by:

Daniela Bettoni,

Osservatorio Astronomico di Padova

(INAF), Italy

Milan S. Dimitrijevic,

Astronomical Observatory, Serbia

*Correspondence:

Ana I. Gomez de Castro

aig@ucm.es

Specialty section:

This article was submitted to

Stellar and Solar Physics,

a section of the journa

Frontiers in Astronomy and Space

Sciences

Received: 05 April 2018

Accepted: 05 July 2018

Published: 03 August 2018

Citation:

Gomez de Castro Al (2018) Is Life an Unavoidable Consequence of the

Formation of the Universe?

Investigating the Formation of Bio-Precursors and the Signature of

Earth-Like Living Forms.

Front. Astron. Space Sci. 5:22.

doi: 10.3389/fspas.2018.00022

\section{Is Life an Unavoidable Consequence of the Formation of the Universe? Investigating the Formation of Bio-Precursors and the Signature of Earth-Like Living Forms}

\author{
Ana I. Gomez de Castro* \\ AEGORA Research Group, Universidad Complutense, Fac. CC Matematicas, Madrid, Spain
}

This contribution to the Research Topic "Imagining the Future of Astronomy and Space Sciences" focuses on astrobiology and exoplanetary research. Understanding the origin of life is the main scientific challenge to this century and an interdisciplinary endeavor in itself. To that astronomy will contribute in three key issues. Firstly, by measuring the abundance of elements relevant to life in the Universe. Then by determining the preferred location for aminoacids and complex organic molecules assembly. Finally, by investigating the signatures of life in exoplanets. A new generation of facilities will need to be built to address these questions. The relevance of ultraviolet instrumentation for this purpose is highlighted in this short perspective.

Keywords: astrobiology, astronomy, ultraviolet, exoplanets, chemical evolution

\section{INTRODUCTION}

Life is plenty in planet Earth but whether this phenomenon is ubiquitous in the Galaxy and sustainable over time scales comparable to stellar evolution is a big unknown. Our century is born in the confidence that these big questions can be addressed with our current scientific understanding of nature and the technology to be developed in the near future. Astronomy will contribute to this endeavor in three key issues: measuring the chemical evolution of the Universe, finding the sites where life can be formed and seeking for evidence of life outside the Earth.

The chemical evolution of the Universe and the requirements, in terms of carbon, nitrogen, oxygen, sulfur and phosphor (CNOSP) abundances, for life to be sustained needs to be investigated by astronomers. For instance, virtually all forms of life on Earth use adenosine trisphosphate $\left(\mathrm{C}_{10} \mathrm{H}_{16} \mathrm{~N}_{5} \mathrm{O}_{13} \mathrm{P}_{3}\right)$ for energy exchange and storage however, all these elements unless hydrogen result from the chemical evolution of the Universe. Important questions to be answered are: could life be generated in other regions or times along the history of the Universe? Where and when should the signatures of life be looked for?

Then, it is necessary to investigate the location where aminoacids and complex proteins are assembled. Glycine, the simplest and most abundant aminoacid has been detected in comets. Are aminoacids formed in comets and rain down on the dry planetary surfaces or do they form directly on planets? Which are the requirements to build proteins from scratch? Is any of these requirements associated with the stellar evolution? For instance, could life be generated as early as in the planetesimal formation phase? 
Finally, a broader perspective on Earth studies is required, Earth needs to be investigated as an exoplanet. In the quest for extraterrestrial life, Earth is the only reference henceforth its electromagnetic signature, including polarimetric information, needs to be characterized in full detail. This will allow addressing the key questions: which is the imprint of life on the signal received from exoplanets? And of intelligent life?

Major investments are being made to seek for Earth analogs in space and to investigate the chemical processes behind the origin of life. Ultraviolet astronomy is a key player in this task. In this article, the scientific rationale behind this statement will be reviewed (section Ultraviolet astronomy in the quest for the origin of life), as well as the actions being taken for the implementation of the required instrumentation (section On-going activities to develop UV instrumentation). A short summary and brief conclusions are provided at the end (section Summary and conclusions).

\section{ULTRAVIOLET ASTRONOMY IN THE QUEST FOR THE ORIGIN OF LIFE}

Investigating the origin of life means determining when and how the building blocks of life are assembled. Building blocks are: elements, molecules, aromatic hydrocarbons, aminoacids, and RNA. The laws of Physics establish the possible combinations of neutrons, electrons and protons leading to stable elements however, their abundance in nature depends on the specifics of some astrophysical processes: stellar nucleo-synthesis, supernovae explosions and gas mixing in the Galaxy, yet not fully understood.

Life on Earth grows from given (solar) abundance of elements. Could life be generated in other regions or times along the history of the Universe?

For instance, would a drop by 1 dex in the phosphorus abundance $^{1}$ prevent life formation? (Gibard et al., 2018). Life without Phosphor, at least on Earth is impossible, because all major building blocks of life contain phosphate groups, including ATP (the energy carrier of life), RNA, DNA, and cellular membranes. The answer to this question will come from biochemistry laboratories; adenosine triphosphate (ATP) is a complex organic molecule found in all forms of life on Earth. However, once a threshold is established, the possible locations and times in the history of the Universe where ATP can be formed will have to be determined by astronomers.

Phosphorus is believed to be mainly formed in massive stars in the pre-SN stage during the carbon and neon burning in a hydrostatic shell (Woosley and Weaver, 1995). There are however, uncertainties in the details of the process. The scarce measurements carried out with the Copernicus satellite, the Far Ultraviolet Spectroscopic Explorer (FUSE) and the Hubble Space Telescope are consistent with a solar-like abundance of phosphorus in the diffuse (nearby) interstellar medium (Dufton et al., 1986; Lebouteiller and Ferlet, 2005). However, the abundance of phosphorus in the Cass A supernova remnant

${ }^{1}$ The solar abundance of Phosphorus is $1.16 \times 10^{-2}$ times the abundance of iron by number (Anders and Grevesse, 1989). is about 100 times that observed in the ISM (Koo et al., 2013) thus, mixing processes must play a fundamental role in the final chemical budget.

Resonance transitions provide the best chances to measuring the distribution of Phosphorus in space and testing these models; Phosphorus is the least abundant of the elements required for life $\mathrm{H}, \mathrm{C}, \mathrm{N}, \mathrm{O}, \mathrm{S}, \mathrm{P}$ (H CNOSP) and its detection requires using the most sensitive tracers. For P I and P II, the resonance transitions are in the $120-170 \mathrm{~nm}$ range (in the ultraviolet).

In fact, this is not an uncommon case. The ultraviolet (UV) range is unmatched by any other spectral range in number of resonance transitions of $\mathrm{H}$ CNOSP elements, as shown in Figure 1. At temperatures below $30,000 \mathrm{~K}$, more than $75 \%$ of the resonance transitions of the neutral and singly ionized $\mathrm{H}$ CNOSP are in the $90-320 \mathrm{~nm}$ range, with the only exception of nitrogen. If all possible ionization states are included, still about a $30 \%$ of all the resonance transitions are in the $90-320 \mathrm{~nm}$ range that covers barely $9.9 \mathrm{eV}$ in energy (from 13.6 to $3.87 \mathrm{eV}$ ).

\section{The Missing Metals Problem}

All elements heavier than Boron are produced within galaxies by stars and stellar by-products such as supernovae explosions or massive post-AGB outflows. However, only $\sim 20 \%$ of all metals produced in galaxies are retained within. The metal mass densities inferred from damped Lyman- $\alpha$ (Ly $\alpha)$ systems at $\mathrm{z} \in[0.5,5]$ are a factor of 10 lower than expected from the star formation history of the universe (Prochaska et al., 2003). Therefore, there is a "missing metals problem" that needs to be addressed.

Following (Peeples et al., 2014) calculations, roughly 80-85\% of the metals (by mass) are produced in core-collapse supernovae; the rest is produced in Type Ia supernovae explosions and by post-AGB stars. Hence, the expected metallicity of any given galaxy can be predicted from its star forming history. However, the values inferred from the theoretical modeling exceed by a factor of $\sim 4$ the total inventory of metals, including metals trapped in stars and those released to the interstellar medium (ISM) either in the form of gas or dust (see Figure 2 as in Peeples et al., 2014).

As a result, the onset of highly efficient galactic winds needs to be invoked to remove the freshly produced metals from the ISM and send it into the circumgalactic space. In the halo of normal galaxies at $\mathrm{z}<1$, chemically enriched material with metallicities $\sim 50 \%$ solar is ejected by galactic winds. However, also metal poor gas (metallicity $\sim 4 \%$ solar) is detected in a roughly $1: 1$ proportion (Wotta et al., 2016) probably accreting material, either from the galactic corona or from the intergalactic space. There seems to be a sequence with time (redshift) to higher metallicities and cleaner separation between the two components as present is approached in the lifetime of the Universe (Lehner et al., 2016; Tumlinson et al., 2017). According to numerical simulations the enrichment of the hot circumgalactic medium proceeds in an "inside-out" fashion throughout the evolution of galaxies: metals are transported from the original (main central) galaxy by supernova-driven winds over several Gyr, producing a strong radial metallicity gradient (Crain et al., 2013). Critical tests to these models require measuring the physical extent of metals 


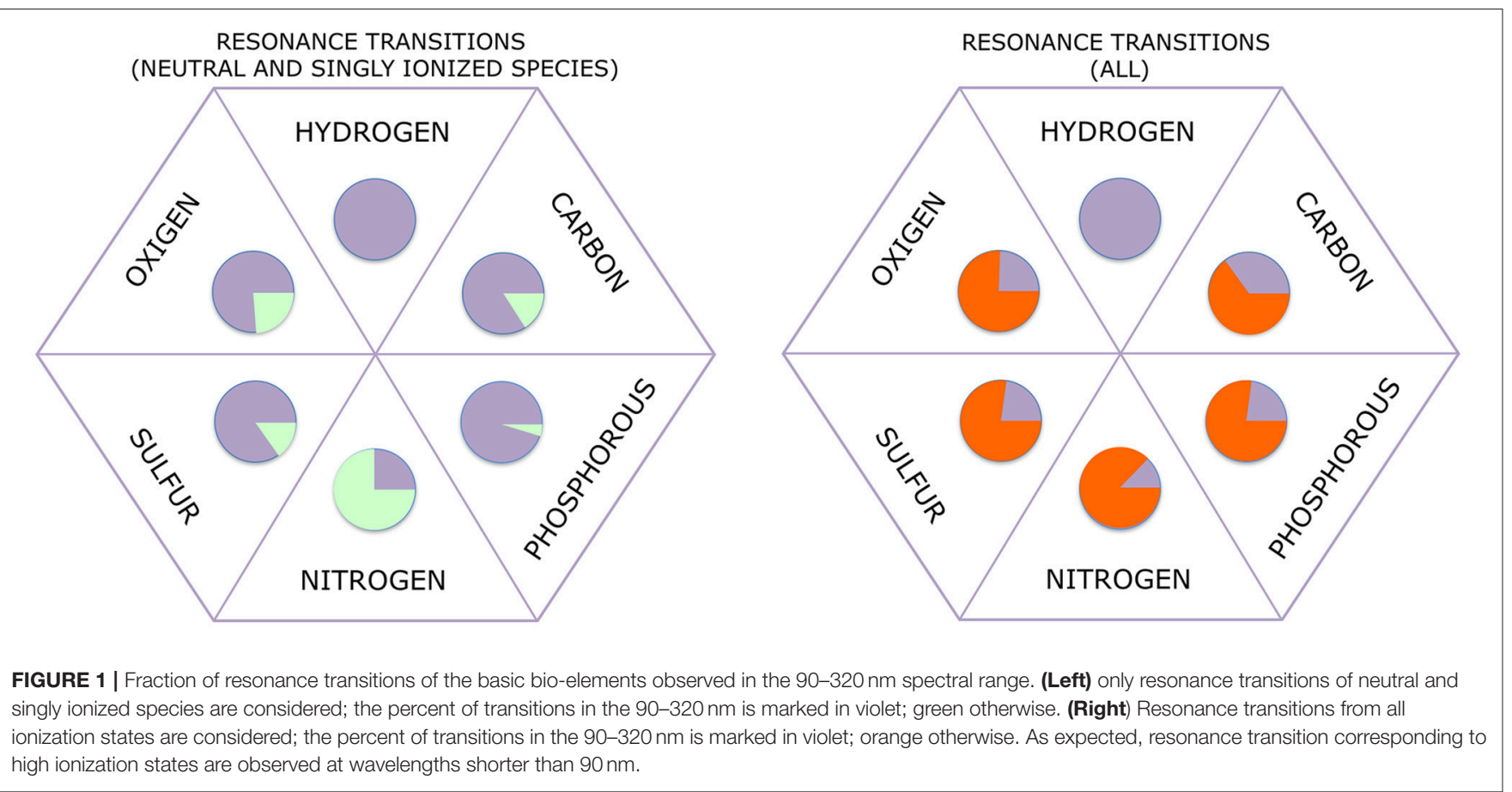

around galaxies, and their relative mixtures in the phases of the gas. This will need to be done in the UV.

As corollary for bio-astronomical research, astronomical evidence points toward a slower pace in the chemical enrichment of the Universe than previously thought; a fraction of the ISM metals get lost to the diffuse intergalactic space instead of enriching the next stellar generation.

\section{The Earth's UV Signature}

As today, Earth is the only reference to qualify a living planet. For remote sensing purpose, life most prominent signature comes from the atmosphere and its trace is noticeable across the electromagnetic spectrum. Detectability is difficult because of the intrinsic weakness of the planetary signal compared to the star. For these reason, observation of planetary transit is the preferred and more efficient technique to detect and characterize exoplanets, though currently several experiments are being planned to resolve planets from their parent star (see section On-Going Activities to Develop UV Instrumentation).

Observing planetary transits gives access to important properties of the planets and their interaction with the parent star (Vidal-Madjar et al., 2003; Kulow et al., 2014). The depth and shape of the transit light curve is the most straightforward observable and the most successful detection method: $\sim 77 \%$ of the exoplanets known today have been detected by transits. Spectroscopic observations of transits have been feasible only for hot Jupiters (giant gaseous planets in close orbit around the star) and the light curves are found to depend on the spectral tracer (Charbonneau et al., 2002; Vidal-Madjar et al., 2004), providing important clues on planetary exospheres and photo-evaporative processes; the Lyman- $\alpha$ (Ly $\alpha)$ line, at $122 \mathrm{~nm}$, is the strongest feature in the spectrum and provides the cleanest signatures.

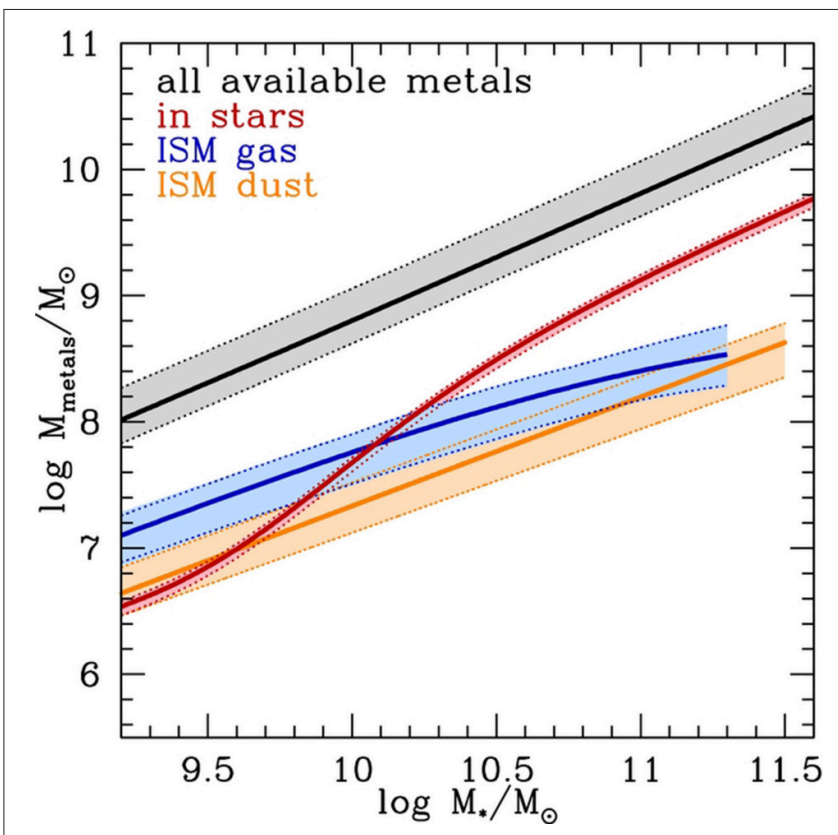

FIGURE 2 | Total mass of metals produced by supernovae and AGB stars (black), the mass of metals currently in stars (red), the mass of metals in interstellar gas (blue), and the mass of metals in dust (orange), all plotted vs. the $z=0$ galactic stellar mass of star-forming galaxies. The shaded regions and dotted lines denote the adopted uncertainties (after Peeples et al., 2014, reproduced by permission of the AAS).

Detailed calculations have been carried out to evaluate the instrumentation needed to detect bio-tracers in Earth-like planets by the transit method concluding that collecting surfaces 
equivalent to a $10-20 \mathrm{~m}$ primary are required for these new generation of telescopes (Ehrenreich et al., 2006; Gómez de Castro et al., 2006; Arnold et al., 2014). The detection of the $\mathrm{H}$ I envelopes of Earth-like exoplanets may prove to be easier. Observations of the Earth's exosphere have unveiled an extended envelope of hydrogen reaching further than 38 Earth radii $^{2}$ composed of atoms orbiting around the Earth. This large envelope increases significantly the opacity of the Earth to Lya photons coming from the Sun, to the point of making feasible the detection of the Earth's transit signature from barely $1.35 \mathrm{pc}$, the distance to $\alpha$-Cen, if pointing with an 8 -m primary mirror space telescope through a clean line of sight $\left(\mathrm{N}_{\mathrm{H}}<10^{17}\right.$ $\mathrm{cm}^{-2}$ ). Gómez de Castro et al. (2018) show that in spite of the interstellar, heliospheric and astrospheric absorption, the Earthlike exoplanets' transit signature in M5V type stars would be detectable with dedicated Ly $\alpha$ flux monitors implemented in 4$8 \mathrm{~m}$ class space telescopes; some examples of Ly $\alpha$ light curves are displayed in Figure 3. Such monitoring programs would enable measuring the robustness of planetary atmospheres under heavy space weather conditions like those produced by M-type stars. A 2-m class telescope, such as the World Space Observatory, would suffice to detect an Earth-like planet orbiting around Proxima Centauri, if there was such a planet, or nearby M5 type stars.

The Earth atmosphere scatters back into space the UV radiation received from the Sun leaving its imprint on it. Solar radiation impinging the Earth's atmosphere is not polarized but the reflected radiation is polarized. In exoplanetary research, this polarized signal may be easier to discern during transits than the raw photometric signal (Fauchez et al., 2017) providing important clues on the distribution of aerosols and clouds. At UV wavelengths, the Earth's ozone blocks any UV information coming from below $40 \mathrm{~km}$ above the surface, as a result, the polarization of the backscattered signal is dominated by particles and aerosols in the uppermost layers of the Earth's atmosphere, the Earth-Space interface. The solar backscattered UV (SBUV) radiation has a pronounced decrease between 210 and $320 \mathrm{~nm}$ due to absorption by the ozone layer. Below $210 \mathrm{~nm}$, Rayleigh scattering of the solar spectrum by the Earth's atmosphere dominates. The signal drops by 6 orders of magnitude in the 150 $300 \mathrm{~nm}$ range producing a clean feature susceptible to be detected in the UV polarization signature produced by exoplanets.

\section{ON-GOING ACTIVITIES TO DEVELOP UV INSTRUMENTATION}

As today, there is only one $2-\mathrm{m}$ class observatory working in the UV range, the Hubble Space Telescope that has been operational since 1990 at low Earth orbit. There are also some few small ${ }^{3}$ facilities operating in the UV range namely, the Ultraviolet and Optical Telescope (UVOT), on-board the SWIFT satellite, the Optical Monitor on the XMM-Newton X-ray observatory and the Ultraviolet Imaging Telescope (UVIT), on-board the Astrosat satellite. Moreover, there is a small (15-cm aperture) telescope

\footnotetext{
${ }^{2}$ Recent data obtained by the Japanese Space Agency (JAXA) point toward an exospheric radius $>38 \mathrm{R}_{\mathrm{E}}$ ! (Shingo Kameda, private communication).

${ }^{3}$ Equivalent to a $10-40 \mathrm{~cm}$ diameter circular aperture.
}

on the Moon, the Lunar Ultraviolet Telescope (LUT), operating from China's lunar lander Chang'e 3 launched in December 2013; LUT is fully operational and could work for 30 years.

As Hubble operational lifetime is approaching the end, the astronomical community is coordinating to develop large UV telescopes with effective area at least 2 orders of magnitude larger than Hubble to get deeper into the intergalactic space, study the atmospheres of the known exoplanets and seek for evidence of life. These large facilities must be able to address these crucial problems while allowing observatory type operations to enable the broad diversity of science to develop and optimize the scientific return of the heavy expenditures. There have been several concepts circulating during the last two decades to unify behind a single, very large collecting surface, a set of instruments covering the 90-3,000 $\mathrm{nm}$ spectral range, i.e., to reproduce (and enhance) Hubble's capabilities from the far UV to the near infrared.

In the United States of America, the first proposals were driven by the past decade planning. ST-2010 was a mission concept to a 4-8 m UV-Optical space telescope (Shull, 1999). The Modern Universe Space Telescope (MUST) proposal consisted of a 10-m telescope equipped with spectroscopic instruments for multi-object observations and integral field spectroscopy with high multiplexing capabilities to produce up to a thousand of simultaneous spectra in a data cube. The Advanced Technology Large-Aperture Space Telescope (ATLAST) was a set of mission concepts for observatories with a primary aperture diameter in the $8-16 \mathrm{~m}$ range (Postman et al., 2009). For the coming decadal review, these concepts have converged into the Large UVOptical-Infrared observatory (LUVOIR). The LUVOIR concept (Bolcar et al., 2017) stands for a large, serviceable observatory with a deployable primary mirror that comes in two possible architectures: Architecture A (15.1-m mirror) that will require launching with NASA's planned Space Launch System (SPS) and Architecture B (9.2-m mirror) designed to be launched with a heavy-lift launch vehicle, similar to those in use today. LUVOIR will be competing with other three projects to become the NASA's flagship at the 2020 decadal survey.

In Europe, the call issued by the European Space Agency to raise the scientific drivers for the next generation of large missions inspired the EUVO proposal to build the European Ultraviolet-Visible Observatory (Gómez de Castro et al., 2014). The baseline design was a deployable 8 -m space telescope providing a factor 100 improvement in sensitivity compared with Hubble. The outlined technical concept was similar to LUVOIR Architecture-B. A summary of the scientific requirements from the European astronomical community to EUVO is in Table 1. European astronomers have also coordinated behind the French Space Agency (CNES) to propose an instrument for high dispersion spectropolarimetry in the $98-390 \mathrm{~nm}$ spectral range with LUVOIR named POLLUX (Bouret et al., 2018).

The short UV wavelengths are ideally suited for high resolution imaging since angular resolution ${ }^{4}$ is proportional to wavelength for a given aperture size. The Fresnel Imager (FI)

${ }^{4}$ For a circular aperture, angular resolution is given by $\theta \approx 1.22 \lambda / \mathrm{D}$ with $\lambda$ the radiation wavelength and $\mathrm{D}$ the diameter of a circular aperture. 

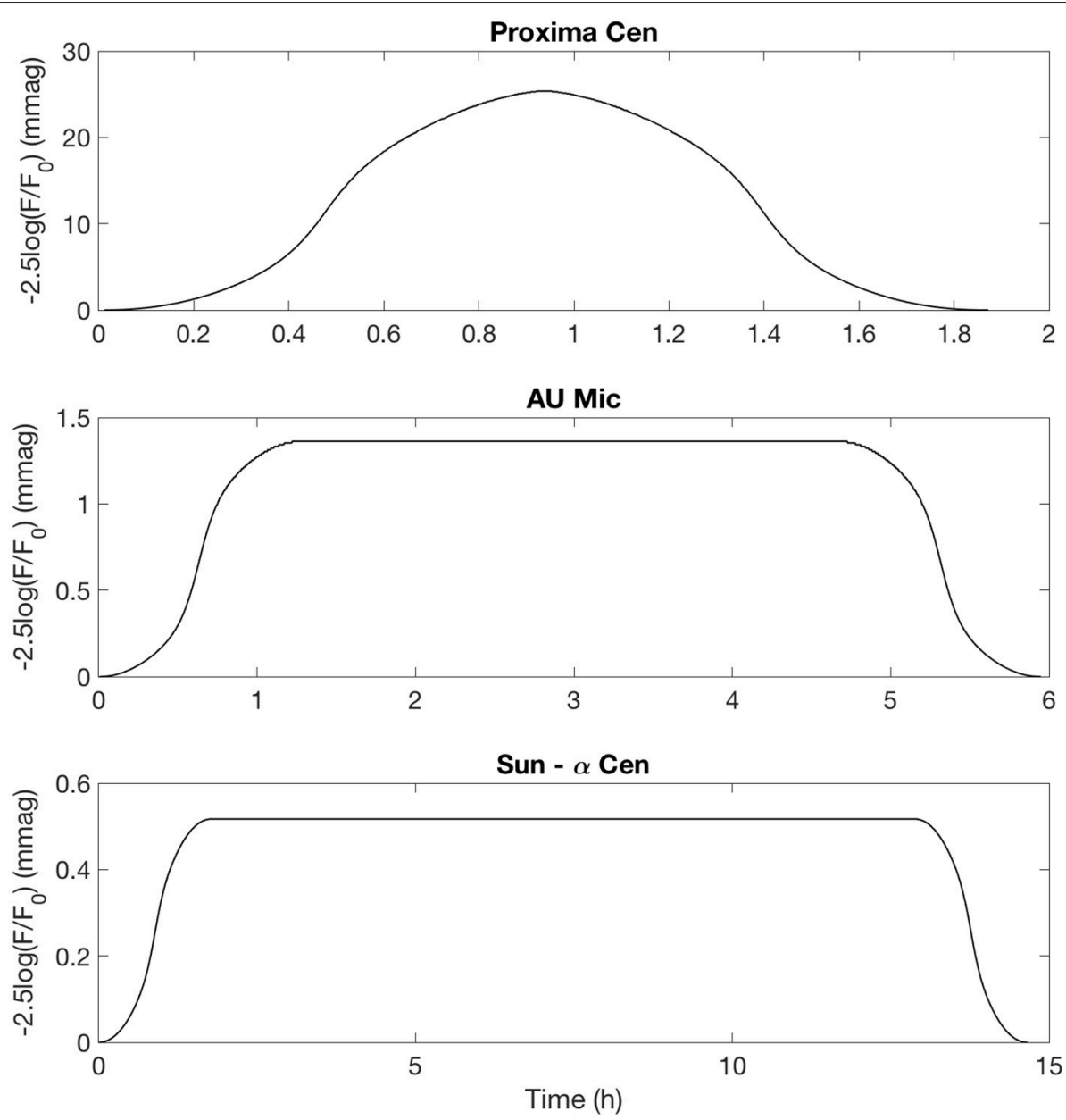

FIGURE 3 | Theoretical Lya light curves simulating the transit of an Earth-like exoplanet with an extended exosphere, alike the Earth's one. Calculations were made for the transit of such a planet, orbiting in the habitable zone around an M5V (Proxima Cen), an M0V (AU Mic) and the Sun. For the model, it is assumed that $90 \%$ of the transit signal is absorbed by thin interstellar clouds (see Gómez de Castro et al., 2018 for details).

makes use of this advantage to resolve and image exoplanets and, in general, weak structures close to bright sources requiring high dynamical range and diffraction limited resolution (Koechlin et al., 2009). Per design, the FI is a two spacecraft formationflying instrument, one spacecraft holds the interferometric array acting as focusing element; the other spacecraft holds the optical payload including the instruments. A $3.6 \times 3.6 \mathrm{~m}$ square opaque foil punched with about 10,000 "subapertures" suffices to produce a diffraction limited image at UV wavelengths with dynamical range $10^{-8}$. FI concept is different than the proposed one for the Habitable Exoplanets Imaging mission (HabEx) competing with LUVOIR at the 2020 decadal survey. HabEx is investigating two methods: a coronographic instrument within the telescope and a starshade (Stark et al., 2016). The starshade is a large petal shaped vehicle which blocks light before entering the telescope and requires formation flying as the FI does. From the optics point of view, FI forms image by diffraction which is a natural approach for space-based observatories having as main handicap the poor radiometric efficiency. Starshades however, work like external coronagraphs.
FIs can work simultaneously from the 0.1 to few microns and accommodate separate optics for the various ranges in independent vessels. HabEx starshade is designed to work from 0.55 to 1 micron wavelength.

If successful, these projects will be flown in the early 40's, far in the future. As today, the only 2-m class observatory in development is the World Space Observatory-Ultraviolet (WSO-UV) to be launched in few years (Shustov et al., 2018). WSO-UV is the international name of Spectrum-UV, the observatory of the series Spectrum being part of the scientific program of the Russian Space Agency. Spectrum-UV is a collaborative project between Russia and Spain and will be equipped with instrumentation similar to Hubble and operated in observatory mode. Though the sensitivity of HST/COS will be unmatched by WSO-UV/WUVES in the $115-170 \mathrm{~nm}$ range where the resonance transitions of $\mathrm{CI}$, OI, HI reside, WSOUV/WUVES would be equipped with new technology detectors that will grant a sensitivity about 10 times that of HST/COS or HST/STIS in the $220-310 \mathrm{~nm}$ range where the $\mathrm{OH}, \mathrm{CS}$, $\mathrm{C}_{2}$ and polycyclic aromatic hydrocarbons (PAHs) bands reside 
TABLE 1 | Scientific requirements for EUVO from Gómez de Castro et al. (2014).

\begin{tabular}{|c|c|c|}
\hline \multicolumn{2}{|l|}{ Requirement } & \multirow{2}{*}{$\begin{array}{l}\text { Requirement science topic } \\
\text { Dark sky to study faint sources } \\
\text { Variability studies in all topics (AGNs, stellar astrophysics, Solar System Research) }\end{array}$} \\
\hline L2 orbit (or high Earth Orbit) & & \\
\hline Large focal planes & $\begin{array}{l}\text { field of view: } \\
10 \times 10 \text { arcmin, } \\
\text { angular resolution: } \\
<0.01 \text { arcsec }\end{array}$ & Efficient instrumentation for galactic and extragalactic surveys \\
\hline Spectral coverage & 90-320 nm & From the Lyman continuum to the Werner H2 bands for ISM and IGM studies, TTSs disks, planetary aurorae \\
\hline Integral field spectroscopy & $R=500-3,000$ & $\begin{array}{l}\text { Efficient instrumentation to characterize astronomical sources in surveys. } \\
\text { Narrow band imaging in the FUV of extended structures (jets, nebulae,star forming regions, clusters) }\end{array}$ \\
\hline Spectroscopy & $R=10,000$ & IGM and galactic haloes observations \\
\hline & $R=20,000$ & Young stars and stellar astrophysics, in general \\
\hline & $\mathrm{R}=80,000$ & Stellar astrophysics \\
\hline & $R>100,000$ & $\begin{array}{l}\text { ISM studies and fine structure constant measurements and dynamical range }>>100 \text { for research in exoplanets } \\
\text { auroral activity. }\end{array}$ \\
\hline Spectropolarimetry & $\begin{array}{l}R=10,000 \\
R=80,000\end{array}$ & $\begin{array}{l}\text { Young stars (polarization accuracy }<0.1 \% \text { ) } \\
\text { Stellar astrophysics (polarization accuracy < } 0.1 \% \text { ) }\end{array}$ \\
\hline Sensitivity ${ }^{\mathrm{a}}$ & $\begin{array}{l}1-10 \\
100\end{array}$ & $\begin{array}{l}\text { All fields of research require to have, at least one, facility working in the UV. Some examples are: } \\
\text { - reaching } 10 \mathrm{Mpc} \text { in the cosmic web research } \\
\text { - studying galactic massive star forming regions such as Orion } \\
\text { - detecting the gas component from transitional to debris disk } \\
\text { - studying the interplanetary medium in exoplanetary systems } \\
\text { - studying M stars within } 50 \mathrm{pc} \\
\text { - observing Uranus as Jupiter is observed with HST }\end{array}$ \\
\hline
\end{tabular}

a Quoted in factors of improvement over similar instrumentation in the HST.

together with the resonance transitions of magnesium and iron. Moreover, WSO-UV/FCU will be the first imager ever flown high above the Earth geocorona, into geosynchronous orbit.

Cubesats are a newcomer in the field ready to stay and grow. The basic cubesat unit $(\mathrm{U})$ is small, " $\mathrm{U}=10 \times 10$ $\times 10 \mathrm{~cm}$ " however the possibility of stacking several (3-12) units together is revolutionizing space science. Typically, a half of a cube is used to incorporate "custom-off-the-shelf" (COTS) navigation and communications components and the rest of the space is left for scientific instrumentation; field optics equivalent to a $40-\mathrm{cm}$ telescope can be accommodated making of cubesats the cheapest and more versatile technology for space research. Though the technology on cubesat formation flying and operation is currently under development, there is still quite a long time to go before it reaches the accuracy to create arbitrarily large collecting surfaces in space. In the meantime, cubesat technology is ideally suited to study the Earth as an exoplanet and small, 6-12 U, have been proposed to measure the interaction between the Earth exosphere and the solar wind (Gómez de Castro et al., in preparation) or to monitor the photoevaporation of hot Jupiters (Fleming et al., 2017).

\section{SUMMARY AND CONCLUSIONS}

Ultraviolet (UV) instrumentation is difficult to build since imposes very stringent requirements to the wave-front control given the short wavelengths. Moreover, it is expensive. Needs to be implemented in space probes. Calibration and performance verification have to be performed in vacuum chambers for the shortest wavelengths. Manufacture of every element and their assembly needs to be carried under conditions of very high cleanness; a layer of molecular deposits of $1.5 \mathrm{~nm}$ thickness produces a reflectivity degradation of a $10 \%$ at Ly $\alpha$. However, $U V$ instrumentation is required for the investigation of the origin of life in the Universe. Studying the chemical evolution, determining the preferred location for aminoacids and complex organic molecules assembly, investigating the signatures of life in exoplanets, all require of UV observations.

A large UV mission with effective area about a hundred times that of Hubble is required to image and characterize the nearest Earth-like exoplanets and seeking for evidence of life in them. In will take about 20 years to raise the funding and develop such mission. In the meantime, the 2-m class WSO-UV will grant continuity to the research initiated with Hubble on the chemical evolution of the nearby Universe and the formation of planetary systems. Small and cubesat sized missions will provide versatile instrumentation to characterize the Earth and study the brightest end of the exoplanetary population.

\section{AUTHOR CONTRIBUTIONS}

The author confirms being the sole contributor of this work and approved it for publication. 


\section{FUNDING}

This work has been partially funded by the Spanish Ministry of Economy, Industry and Competitiveness through grant: ESP2015-68908-R.

\section{REFERENCES}

Anders, E., and Grevesse, N. (1989). Abundances of the elements - meteoritic and solar. Geochim. Cosmochim. Acta 53, 197-214.

Arnold, L., Ehrenreich, D., Vidal-Madjar, A., Dumusque, X., Nitschelm, C., Querel, R. R., et al. (2014). The Earth as an extrasolar transiting planet. II. HARPS and UVES detection of water vapour, biogenic O2, and O3. Astron. Astrophys. 564:58. doi: 10.1051/0004-6361/201323041

Bolcar, M. R., Aloezos, S., Bly, V. T., Collins, C., Crooke, Dressing, C. D., et al. (2017). The Large UV/Optical/Infrared Surveyor (LUVOIR): decadal mission concept design update. Proc. SPIE 10398:1039809. doi: 10.1117/12.2273848

Bouret, J. C., Neiner, C., Lopez Ariste, A., Vivés, S., Muslimov, E., Lopes, L., et al. (2018). POLLUX: a UV High-resolution spectropolatimeter for LUVOIR. Am. Astron. Soc.

Charbonneau, D., Brown, T. M., Noyes, R. W., and Gilliland, R. L. (2002). Detection of an extrasolar planet atmosphere. Astrophys. J. 568:377. doi: $10.1086 / 338770$

Crain, R. A., McCarthy, I. G., Schaye, J., Theuns, T., and Frenk, C. S. (2013). Enriching the hot circumgalactic medium. Mon. Not. R. Astron. Soc. 432, 3005-3024 doi: 10.1093/mnras/stt649

Dufton, P. L., Keenan, F. P., and Hibbert, A. (1986). The abundance of phosphorus in the interstellar medium. Astron. Astrophys. 164, 179-183.

Ehrenreich, D., Tinettz, G., Lecavelier des Etangs, A., Vidal-Madjar, A., and Selsis, F. (2006). The transmission spectrum of Earth-size transiting planets. Astron. Astrophys. 448:379. doi: 10.1051/0004-6361,20053861

Fauchez, T., Rossi, L., and Stam, D. M. (2017). The O2 a-band in the fluxes and polarization of starlight reflected by earth-like exoplanets. Astrophys. J. 842:41. doi: 10.3847/1538-4357/aa6e53

Fleming, B. T., France, K., Nell, N., Kohnert, R., Pool, K., and Egan, A., et al. (2017). The Colorado Ultraviolet Transit Experiment (CUTE): a dedicated cubesat mission for the study of exoplanetary mass loss and magnetic fields. Proc. SPIE 10397:6138. doi: 10.1117/12.2276138

Gibard, C., Bhowmik, S., Karki, E., Kim, E. K., and Krishnamurthy, R. (2018). Phosphorylation, oligomerization and self-assembly in water under potential prebiotic conditions. Nat. Chem. 10, 212-217. doi: 10.1038/nchem.2878

Gómez de Castro, A. I., Appourchaux, T., Barstow, M. A., Barthelemy, M., Baudin, F., Benetti, S., et al. (2014). Building galaxies, stars, planets and the ingredients for life between the stars. The science behind the European ultraviolet-visible observatory. Astrophys. Space Sci. 354, 229-246. doi: 10.1007/s10509-014-1942-7

Gómez de Castro, A. I., Beitia-Antero, L., and Ustamujic, S. (2018). On the feasibility of studying the exospheres of Earth-like exoplanets by Lyman- $\alpha$ monitoring. Exp. Astron. 45, 147-163. doi: 10.1007/s10686-018-9 574-5

Gómez de Castro, A. I., Lecavelier, A., D’Avillez, M., Linsky, J. L., and Cernicharo, J. (2006). UV capabilities to probe the formation of planetary systems: from the ism to planets. Astrophys. Space Sci. 303, 33-52. doi: 10.1007/s10509-006-8793-9

Koechlin, L., Serre, D., Deba, P., Pelló, R., Peillon, C., and Duchon, P., et al. (2009). The fresnel interferometric imager. Exp. Astron. 23, 379-402. doi: 10.1007/s10686-008-9112-y

Koo, B. C., Lee, Y. H., Moon, D. S., Yoon, S. C., and Raymond, J. C. (2013). Phosphorus in the young supernova remnant cassiopeia A. Science 342, 1346-1348. doi: 10.1126/science. 1243823

Kulow, J. R., France, K., Linsky, J., and Parke Loyd, R. O. (2014). Ly $\alpha$ transit spectroscopy and the neutral hydrogen tail of the hot Neptune GJ 436b. Astrophys. J. 786:132. doi: 10.1088/0004-637X/786/2/132

\section{ACKNOWLEDGMENTS}

This work has been inspired by the activity of the UV Astronomy working group of the European Astronomical Union.

Lebouteiller, V. K., and Ferlet, R. (2005). Phosphorus in the diffuse interstellar medium. Astron. Astrophys. 443:509. doi: 10.1051/0004-6361,200 53448

Lehner, N., O’Meara, J. M., Howk, J. C., Prochaska, J. X., and Fumagalli, M. (2016). The cosmic evolution of the metallicity distribution of ionized gas traced by lyman limit systems. Astrophys. J. 833:283. doi: 10.3847/1538-4357/833/2/283

Peeples, M. S., Werk, J. K., Tumlinson, J., Oppenheimer, B. D., Prochaska, J. X., and Katz, N., et al. (2014). A Budget and accounting of metals at $\mathrm{z} \sim 0$ : results from the COS-halos survey. Astrophys. J. 786:54. doi: 10.1088/0004-637X/786/1/54

Postman, M., Argabright, V., Arnold, B., Aronstein, D., Atcheson, P., Blouke, M., Brown, T., et al. (2009). Advanced Technology Large-Aperture Space Telescope (ATLAST): a technology roadmap for the next decade, RFI submitted to Astro2010 Decadal Committee. arXiv:0904.0941.

Prochaska, J. X., Gawiser, E., Wolfe, A. M., Castro, S., and Djorgovski, S. G. (2003). The age-metallicity relation of the universe in neutral gas: the first 100 damped Lyo systems. Astrophys. J. 595:L9. doi: 10.1086/378945

Shull, J. M. (1999). "Large space ultraviolet observatory ST-2010: initial plans for a moderate-class UV/O mission," in Ultraviolet-Optical Space Astronomy Beyond HST, eds A. Jon, J. Morse, M. Shull, and A. L. Kinney (ASP Conference Series), 229.

Shustov, B., Gómez de Castro, A. I., Sachkov, M., Vallejo, J. C., Marcos-Arenal, P., and Kanev, E., et al. (2018). The world space observatory ultraviolet (WSO-UV), as a bridge to future UV astronomy. Astrophys. Space Sci. 363:62. doi: 10.1007/s10509-018-3280-7

Stark, C. C., Cady, E. J., Clampin, M., Domagal-Goldman, S., Lisman, D., and Mandell, A. M., et al. (2016). A direct comparison of exoEarth yields for starshades and coronagraphs. Proc SPIE 9904:99041U. doi: 10.1117/12. 2233201

Tumlinson, J., Peeples, M. S., and Werk, J. K. (2017). The circumgalactic medium. Ann. Rev. Astron. Astrophys. 55:387. doi: 10.1146/annurev-astro-091916-055240

Vidal-Madjar, A., Désert, J. M., Lecavelier des Etangs, A., Hébrard, G., Ballester, G. E., Ehrenreich, D., et al. (2004). Detection of oxygen and carbon in the hydrodynamically escaping atmosphere of the extrasolar planet HD 209458b. Astrophys. J. 604:L69. doi: 10.1086/383347

Vidal-Madjar, A., Des Etangs, A. L., Désert, J. M., Ballester, G. E., Ferlet, R., Hébrard, G., et al. (2003). An extended upper atmosphere around the extrasolar planet HD209458b. Nature 422, 143-146. doi: 10.1038/nature01448

Woosley, S. E., and Weaver, T. A. (1995). The evolution and explosion of massive stars. II. explosive hydrodynamics and nucleosynthesis, astrophys. J. Suppl. 101:181 doi: 10.1086/192237

Wotta, C. B., Lehner, N., Howk, J. C., O’Meara, J. M., and Prochaska, J. X. (2016). Low-metallicity Absorbers Account for Half of the Dense Circumgalactic Gas at $\mathrm{z}$ ? 1. Astrophys. J. 831:95. doi: 10.3847/0004-637X/831/1/95

Conflict of Interest Statement: The author declares that the research was conducted in the absence of any commercial or financial relationships that could be construed as a potential conflict of interest.

The reviewer DB and handling editor declared their shared affiliation.

Copyright (C) 2018 Gomez de Castro. This is an open-access article distributed under the terms of the Creative Commons Attribution License (CC BY). The use, distribution or reproduction in other forums is permitted, provided the original author(s) and the copyright owner(s) are credited and that the original publication in this journal is cited, in accordance with accepted academic practice. No use, distribution or reproduction is permitted which does not comply with these terms. 